\title{
Association between clinical characteristics and short-term outcomes in adult male COVID-19 patients with mild clinical symptoms: a single- center observational study
}

\section{Bailing Yan}

Jilin University First Hospital

\section{Lei Song}

Jilin University First Hospital

Jia Guo

Jilin University First Hospital

\section{Yangyang Wang}

Jilin University First Hospital

\section{Liping Peng}

Jilin University First Hospital

Dan Li (D li_dan@jlu.edu.cn )

Jilin University First Hospital

\section{Research}

Keywords: COVID-19; chest imaging study; peripheral blood oxygen saturation; observational study.

Posted Date: May 21st, 2020

DOI: https://doi.org/10.21203/rs.3.rs-29526/v1

License: (c) (i) This work is licensed under a Creative Commons Attribution 4.0 International License.

Read Full License 


\section{Abstract}

Background: Majority of patients with 2019 novel coronavirus infection (COVID-19) exhibit mild symptoms. Identification of COVID-19 patients with mild symptoms who might develop into severe or critical illness is essential to save lives.

Materials and Methods: We conducted an observational study in a dedicated make-shift hospital for adult male COVID-19 patients with mild symptoms between February and March 2020. Baseline characteristics, medical history, and clinical presentation were recorded. Laboratory tests and chest computed tomography were performed. Patients were observed until they were either transferred to a hospital for advanced care owing to disease exacerbation or were discharged after improvement. Patients were grouped based on their chest imaging findings or short-term outcomes.

Results: A total of 125 COVID-19 patients with mild symptoms were enrolled. Of these, 7 patients were transferred for advanced care while 118 patients were discharged after improvement and showed no disease recurrence during an additional 28-day follow-up period. Eighty-five patients $(68.0 \%)$ had abnormal chest imaging findings. Patients with abnormal chest imaging findings were more likely to have disease deterioration and require advanced care as compared to those with normal chest imaging findings. Patients with deteriorated outcomes were more likely to have low peripheral blood oxygen saturation and moderately-elevated body temperature. There were no significant differences between patients with deteriorated or improved outcomes with respect to age, clinical presentation, or comorbidities.

Conclusions: Abnormal chest imaging findings, low peripheral blood oxygen saturation, and elevated temperature were associated with disease deterioration in adult male COVID-19 patients with mild clinical symptoms.

Trial registration: ClinicalTrials.gov ID: NCT04346602. Registered 12 April 2020- Retrospectively registered, https://register.clinicaltrials.gov/prs/app/action/SelectProtocol? sid=S0009RA3\&selectaction=Edit\&uid=U0003F4L\&ts=2\&cx=-ajpsbw

\section{Introduction}

In December 2019, an outbreak of novel coronavirus pneumonia was reported in Wuhan, China. Subsequently, the outbreak rapidly spread in China and across the world[1]. The World Health Organization (WHO) named this new coronavirus as "2019 Novel Coronavirus (2019-nCoV)"[2]. This novel coronavirus belongs to the same family as the 2003 severe acute respiratory syndrome coronavirus (SARS-CoV). The human disease caused by this novel coronavirus is referred to as COVID-19, which was declared as a pandemic by WHO[3]. As of May 3 2020, the cumulative number of confirmed COVID-19 cases has surpassed 3 million globally, with more than 200,000 deaths reported. The ongoing pandemic has imposed an enormous burden on the society and individual patients. Based on the clinical presentation, laboratory investigations, and imaging findings, patients with COVID-19 can be classified 
into mild, moderate, severe, or critical illness[4]. Several studies have investigated the epidemiology, pathogenesis, and the management of COVID-19. However, most of these studies involved hospitalized patients with moderate or critical illness[5-8]. Few studies have investigated patients with mild or moderate illness, despite the fact that more than $80 \%$ of patients with COVID-19 exhibit mild or moderate illness[9]. It is important to identify patients who have mild initial presenting symptoms, but who are at high risk of developing severe illness. This would help optimize the use of medical resources and reduce the associated morbidity and mortality.

In China, all patients diagnosed with COVID-19 were admitted to a dedicated healthcare facility. This was done to provide centralized care for infected patients and to minimize the risk of community spread of the virus[10]. Patients with mild symptoms were admitted to makeshift hospitals referred to as mobile cabin hospitals; these temporary hospitals were typically set up at sports stadiums or convention centers $[11,12]$. This provided us an opportunity to study the clinical characteristics and short-term outcomes of COVID-19 patients who had mild initial presenting symptoms.

Here, we report the clinical characteristics COVID-19 patients with mild initial presenting symptoms. We further studied the association between the short-term outcomes of these patients and their chest imaging findings and laboratory indices.

\section{Materials And Methods}

\section{Study design and participants}

We performed a prospective observational study at a mobile cabin hospital dedicated for treatment of adult male COVID-19 patients with mild symptoms in Wuhan, China between February and March 2020. The study protocol was approved by the hospital ethics committee. Written informed consent was obtained from all subjects prior to their enrolment.

The inclusion criteria were male adult patients with positive nucleic acid test for SARS-CoV and mild symptoms of upper respiratory tract infection (such as nasal congestion, sore throat, and mild dry cough). Patients with severe symptoms, such as shortness of breath, high fever, or severe diarrhea were excluded from this study.

\section{Study protocol}

Baseline demographic information and medical history were collected from the subjects at the time of admission. Their clinical presentation was recorded. Laboratory tests included complete blood counts, serum biochemistry, and hepatic and renal function tests. All patients also underwent chest computed tomography (CT). 
During their stay at the mobile cabin hospital, patients were closely monitored, including daily physical examination, recording of vital parameters, and pulse oximetry. This was continued until they were either transferred to a hospital for advanced care due to deterioration of their illness or were discharged home after clinical improvement. The criteria for transfer to an advanced care hospital were: 1) exacerbation of any underlying disease, such as hypertension, diabetes, or other illnesses; 2) persistent fever; 3 ) shortness of breath; 4) oxygen saturation $<93 \%$ (by pulse oximetry). The criteria for discharge were: 1) resolution of fever for > 3 days; 2 ) improvement in respiratory symptoms; 3 ) improvement in chest imaging findings; 4) negative nucleic acid test for SARS-CoV-2 in at least two consecutive samples collected more than 24 hours apart.

Patients who were discharged were followed up for an additional 28 days for any signs of disease recurrence.

\section{Statistical analysis}

Continuous data are presented as mean \pm standard deviation or as median and interquartile range (IQR), as appropriate. Categorical data are presented as frequencies and percentages. Patients were grouped based on their chest imaging findings or short-term outcomes. Comparisons between patients with normal or abnormal chest CT findings as well as between patients with deteriorated or improved outcomes were performed using the sum rank test or Chi squared analysis (Fisher exact test), as appropriate. All statistical analyses were performed using SPSS (IBM, USA). $P$ values $<0.05$ were considered indicative of statistical significance.

\section{Results}

A total of 309 patients were admitted to our mobile cabin hospital during the study reference period. Of these, 125 patients qualified the inclusion criteria and were enrolled in the study. Complete records pertaining to clinical evaluation and follow-up outcomes were available for all patients. Seven patients were transferred for advanced care due to disease exacerbation. The remaining 118 patients were discharged home and were followed up for 28 days.

All subjects had a recent history of exposure to COVID-19. The median age of patients was 35 years (IQR, 30-49) (Table 1). A large number of patients (82 patients, $65.6 \%$ ) were current smokers. Most common comorbidities were hypertension (8 patients, $6.4 \%$ ) and diabetes (8 patients, $6.4 \%$ ). Two patients (1.6\%) had cough and 2 patients $(1.6 \%)$ had mild shortness of breath. Eight patients $(6.4 \%)$ had fever. Only one patient $(0.8 \%)$ had mild diarrhea. A total of $85(68.0 \%)$ patients had abnormal chest CT findings at admission (Table 2). The most common abnormality was ground-glass opacities in the lung (70 patients, $56.0 \%$ ). A small number of patients had decreased leukocyte counts ( 6 patients, $4.8 \%,<4 \times 10^{9} / \mathrm{L}$ ) and lymphocyte counts (21 patients, $16.8 \%,<1.5 \times 10^{9} / \mathrm{L}$ ). Platelet count was reduced in $15(12 \%)$ patients. There was mild elevation of hepatic enzyme levels. The renal function was intact in all patients. 
Table 1

Clinical characteristics of the study population

\section{Variables}

Age, median (IQR), years

Smoking history, $\mathrm{N}(\%)$

Never smokers

Ex-smokers

Current smokers

Comorbidities, N (\%)

COPD

Diabetes

Hypertension

Hepatitis B

Fever, N (\%)

Highest temperature during hospital stay, N (\%)

$<37.5^{\circ} \mathrm{C}$

$37.5-38.0^{\circ} \mathrm{C}$

$38.1-39.0^{\circ} \mathrm{C}$

$>39.0^{\circ} \mathrm{C}$

$\mathrm{SpO}_{2}$, median (IQR)

Respiratory symptoms during admission, $\mathrm{N}(\%)$

Nasal congestion

Sore throat

Cough

Hemoptysis

Sputum production

Shortness of breath
All patients $(\mathrm{N}=125)$

$35(30-49)$

$38(30.4 \%)$

$5(4.0 \%)$

$82(65.6 \%)$

$14(14.1 \%)$

1

8

8

1

$8(6.4 \%)$

$2(1.6 \%)$

$3(2.4 \%)$

$2(1.6 \%)$

$1(0.8 \%)$

$98(96-100)$

$0(0 \%)$

$0(0 \%)$

$2(1.6 \%)$

$0(0 \%)$

$0(0 \%)$

$2(1.6 \%)$

COPD, chronic obstructive pulmonary disease; $\mathrm{SpO}_{2}$, peripheral oxygen saturation; IQR, interquartile range. 
Variables

Other symptoms, $\mathrm{N}(\%)$

Fatigue

Headache

Nausea or vomiting

Diarrhea

COPD, chronic obstructive pulmonary disease; $\mathrm{SpO}_{2}$, peripheral oxygen saturation; IQR, interquartile range.
All patients $(\mathrm{N}=125)$

$$
0(0 \%)
$$

$0(0 \%)$

$0(0 \%)$

$1(0.8 \%)$ 
Table 2

Chest imaging findings and laboratory indices

\section{Variables}

Chest computed tomography

Total abnormalities, N (\%)

Ground-glass opacity

Local patchy shadowing

Bilateral patchy shadowing

Interstitial abnormalities

Complete blood counts

Leukocyte count

$>10 \times 10^{9} / \mathrm{L}$

$<4 \times 10^{9} / \mathrm{L}$

Lymphocyte count

$<1.5 \times 10^{9} / \mathrm{L}$

Platelet count

$<150 \times 10^{9} / \mathrm{L}$

Hemoglobin level, g/L

Hepatic and renal functions

AST > 40 U/L, N (\%)

AST $>80 \mathrm{U} / \mathrm{L}, \mathrm{N}(\%)$

ALT > $40 \mathrm{U} / \mathrm{L}, \mathrm{N}(\%)$

ALT > $80 \mathrm{U} / \mathrm{L}, \mathrm{N}(\%)$

TB $17.1 \mu \mathrm{mol} / \mathrm{L}, \mathrm{N}(\%)$

Creatinine $\geq 133 \mu \mathrm{mol} / \mathrm{L}, \mathrm{N}(\%)$

Blood biochemistry

Sodium, $\mathrm{mmol} / \mathrm{L}$

Potassium, $\mathrm{mmol} / \mathrm{L}$
All patients $(\mathrm{N}=125)$

$85(68.0 \%)$

$70(56.0 \%)$

$18(14.4 \%)$

$10(8.0 \%)$

$4(3.2 \%)$

$6.4(5.3-7.4)$

$2(2.1 \%)$

$6(4.8 \%)$

$2.0(1.6-2.3)$

$21(16.8 \%)$

$216(172-261)$

$15(12 \%)$

$145(139-152)$

$14(11.2 \%)$

$1(0.8 \%)$

$60(48.0 \%)$

$16(12.8 \%)$

$7(0.56 \%)$

$0(0 \%)$

140.3 (138.4-141.8)

$4.65(4.34-5.0)$

AST, aspartate aminotransferase; ALT, alanine aminotransferase; TB, total bilirubin; CRP, C-reactive protein; LDH, lactose dehydrogenase; CK, creatine kinase. 


\begin{tabular}{|ll|}
\hline Variables & All patients $(\mathbf{N}=125)$ \\
\hline Chloride, $\mathrm{mmol} / \mathrm{L}$ & $100.3(99.1-101.5)$ \\
\hline $\mathrm{CRP} \geq 10 \mathrm{mg} / \mathrm{L}, \mathrm{N}(\%)$ & $10(8.0 \%)$ \\
\hline $\mathrm{LDH} \geq 250 \mathrm{U} / \mathrm{L}, \mathrm{N}(\%)$ & $1(0.8 \%)$ \\
\hline $\mathrm{CK} \geq 200 \mathrm{U} / \mathrm{L}, \mathrm{N}(\%)$ & $4(0.32)$ \\
\hline $\begin{array}{l}\text { AST, aspartate aminotransferase; ALT, alanine aminotransferase; TB, total bilirubin; CRP, C-reactive } \\
\text { protein; LDH, lactose dehydrogenase; CK, creatine kinase. }\end{array}$ \\
\hline
\end{tabular}

\section{Comparison between patients with normal or abnormal chest CT findings}

Out of 125 patients, 40 patients showed normal chest CT findings, while 85 patients exhibited abnormal CT findings. Patients with normal chest CT findings were significantly younger than those with abnormal CT findings. There were no significant between-group differences with respect to baseline characteristics, clinical presentation, or medical history (Table 3 ). Among the laboratory parameters, a greater number of patients in the abnormal CT group had blood leukocyte count lower than $4 \times 10^{9} / \mathrm{L}$ (Table 4). 
Table 3

Comparison of baseline characteristics, medical history, and clinical presentation between COVID-19 patients with normal or abnormal chest CT findings

\begin{tabular}{|c|c|c|c|}
\hline Variables & $\begin{array}{l}\text { Normal CT } \\
(\mathrm{N}=40)\end{array}$ & $\begin{array}{l}\text { Abnormal CT } \\
(\mathrm{N}=85)\end{array}$ & $P$ value \\
\hline Age, median (IQR), years & $33.5(27-42)$ & $40(30-52.5)$ & 0.02 \\
\hline \multicolumn{4}{|l|}{ Smoking history, N (\%) } \\
\hline Never smokers & $15(37.5 \%)$ & $23(27.1 \%)$ & 0.24 \\
\hline Ex-smokers & $2(5.0 \%)$ & $3(3.5 \%)$ & 0.66 \\
\hline Current smokers & $23(57.5 \%)$ & $59(69.4 \%)$ & 0.19 \\
\hline Comorbidities, N (\%) & $6 / 40(15 \%)$ & $8 / 85(9.4 \%)$ & 0.60 \\
\hline COPD & 1 & 0 & 0.32 \\
\hline Diabetes & 3 & 5 & 1.00 \\
\hline Hypertension & 3 & 5 & 1.00 \\
\hline Hepatitis B & 1 & 0 & 0.32 \\
\hline Fever, N (\%) & $0(0 \%)$ & $8(9.4 \%)$ & 0.11 \\
\hline \multicolumn{4}{|l|}{ Highest temperature during hospital stay, N (\%) } \\
\hline$<37.5^{\circ} \mathrm{C}$ & $0(0 \%)$ & $2(2.4 \%)$ & 1.00 \\
\hline $37.5-38.0^{\circ} \mathrm{C}$ & $0(0 \%)$ & $3(3.5 \%)$ & 0.55 \\
\hline $38.1-39.0^{\circ} \mathrm{C}$ & $0(0 \%)$ & $2(2.4 \%)$ & 1.00 \\
\hline$>39.0^{\circ} \mathrm{C}$ & $0(0 \%)$ & $1(1.2 \%)$ & 1.00 \\
\hline $\mathrm{SpO}_{2}$, median (IQR) & $98(97-100)$ & $97(95-100)$ & 0.54 \\
\hline Respiratory symptoms during admission, $\mathrm{N}(\%)$ & & & 1.00 \\
\hline Nasal congestion & $0(0 \%)$ & $0(0 \%)$ & \\
\hline Sore throat & $0(0 \%)$ & $0(0 \%)$ & \\
\hline Cough & $1(2.5 \%)$ & $1(1.2 \%)$ & \\
\hline Hemoptysis & $0(0 \%)$ & $0(0 \%)$ & \\
\hline Sputum production & $0(0 \%)$ & $0(0 \%)$ & \\
\hline
\end{tabular}

$\mathrm{CT}$, computed tomography; $\mathrm{COPD}$, chronic obstructive pulmonary disease; $\mathrm{SpO}_{2}$, peripheral oxygen saturation; IQR, interquartile range. 


\begin{tabular}{|llll|}
\hline Variables & $\begin{array}{l}\text { Normal CT } \\
\mathbf{( N = 4 0 )}\end{array}$ & $\begin{array}{l}\text { Abnormal CT } \\
\mathbf{( N = 8 5 )}\end{array}$ & Pvalue \\
\hline Shortness of breath & $1(2.5 \%)$ & $1(1.2 \%)$ & \\
\hline Other symptoms, $\mathrm{N}(\%)$ & & 00 \\
\hline Fatigue & $0(0 \%)$ & $0(0 \%)$ \\
\hline Headache & $0(0 \%)$ & $0(0 \%)$ \\
\hline Nausea or vomiting & $0(0 \%)$ & $0(0 \%)$ \\
\hline Diarrhea & $1(2.5 \%)$ & $0(0 \%)$ \\
\hline $\begin{array}{l}\text { CT, computed tomography; COPD, chronic obstructive pulmonary disease; } \mathrm{SpO} \mathrm{O}_{2}, \text { peripheral oxygen } \\
\text { saturation; IQR, interquartile range. }\end{array}$ \\
\hline
\end{tabular}


Table 4

Comparison of laboratory indices between COVID-19 patients with normal or abnormal chest CT findings

$\begin{array}{llll}\text { Variables } & \text { Normal CT } & \text { Abnormal CT } & \text { Pvalue } \\ & (\mathrm{N}=40) & (\mathrm{N}=85) & \end{array}$

Complete blood counts

Leukocyte count

$6.1(5.1-7.3)$

$6.5(5.3-7.5)$

0.45

$>10 \times 10^{9} / \mathrm{L}$

$2(5 \%)$

$0(0 \%)$

0.10

$<4 \times 10^{9} / \mathrm{L}$

$0(0 \%)$

$6(7.1 \%)$

0.02

Lymphocyte count

$1.9(1.7-2.2)$

$2.0(1.6-2.5)$

0.26

$<1.5 \times 10^{9} / \mathrm{L}$

$4(10.0 \%)$

$17(20.0 \%)$

0.21

Platelet count

217 (180.8-259.8)

$215(168-268)$

0.93

$<150 \times 10^{9} / \mathrm{L}$

$3(7.5 \%)$

$12(14.1 \%)$

0.38

Hemoglobin level, g/L

$145(139-155)$

$146(139-152)$

0.60

Hepatic and renal functions

\begin{tabular}{lllr} 
AST > $40 \mathrm{U} / \mathrm{L}, \mathrm{N}(\%)$ & $2(5 \%)$ & $12(14.1 \%)$ & 0.22 \\
AST > $80 \mathrm{U} / \mathrm{L}, \mathrm{N}(\%)$ & $0(0 \%)$ & $1(1.2 \%)$ & 1.00 \\
ALT > $40 \mathrm{U} / \mathrm{L}, \mathrm{N}(\%)$ & $19(47.5 \%)$ & $41(48.2 \%)$ & 1.00 \\
$\mathrm{ALT}>80 \mathrm{U} / \mathrm{L}, \mathrm{N}(\%)$ & $5(12.5 \%)$ & $5(5.9 \%)$ & 0.95 \\
\hline TB $17.1 \mu \mathrm{mol} / \mathrm{L}, \mathrm{N}(\%)$ & $2(5 \%)$ & $0(0 \%)$ & 1.00 \\
\hline Creatinine $\geq 133 \mu \mathrm{mol} / \mathrm{L}, \mathrm{N}(\%)$ & $0(0 \%)$ & & 1.00 \\
\hline Blood biochemistry & & $140.3(138.5-142.0)$ & 0.54 \\
\hline Sodium, mmol/L & $139.8(138.2-141.4)$ & 0.18 \\
\hline Potassium, mmol/L & $4.6(4.3-4.9)$ & $4.7(4.4-5.0)$ & 0.96 \\
\hline Chloride, mmol/L & $100.5(99.1-101.4)$ & $100.1(99.1-101.5)$ & 0.10 \\
\hline CRP $\geq 10 \mathrm{mg} / \mathrm{L}, \mathrm{N}(\%)$ & $0(0 \%)$ & $10(11.8 \%)$ & 1.00 \\
\hline LDH $\geq 250 \mathrm{U} / \mathrm{L}, \mathrm{N}(\%)$ & $0(0 \%)$ & $1(1.2 \%)$ & 1.00 \\
\hline CK $\geq 200 \mathrm{U} / \mathrm{L}, \mathrm{N}(\%)$ & $1(2.5 \%)$ & $3(3.5 \%)$ &
\end{tabular}

AST, aspartate aminotransferase; ALT, alanine aminotransferase; TB, total bilirubin; CRP, C-reactive protein; LDH, lactose dehydrogenase; CK, creatine kinase. 


\section{Comparison between patients with deteriorated or improved outcomes}

Based on the outcomes (discharged to home or referred for advanced healthcare), the 125 patients were assigned to improved outcome group (118 patients) or deteriorated outcome group (7 patients). None of the 118 patients who were discharged home showed recurrence of their clinical symptoms during the 28day follow-up period.

Compared with the improved outcome group, patients in the deteriorated group were more likely to develop fever (Table 5). There were no other significant between-group differences with respect to age, clinical presentation, or medical history. 
Table 5

Comparison of baseline characteristics, clinical presentation, and medical history between COVID-19 patients with deteriorated or improved outcomes

\begin{tabular}{|c|c|c|c|}
\hline Variables & $\begin{array}{l}\text { Deteriorated } \\
\text { outcome } \\
(\mathrm{N}=7)\end{array}$ & $\begin{array}{l}\text { Improved } \\
\text { outcome } \\
(\mathrm{N}=118)\end{array}$ & $\begin{array}{l}P \\
\text { value }\end{array}$ \\
\hline Age, median (IQR), years & $43(25-53)$ & $30(35-49)$ & 0.92 \\
\hline \multicolumn{4}{|l|}{ Smoking history, N (\%) } \\
\hline Never smokers & $2(28.6 \%)$ & $36(30.5 \%)$ & 1.00 \\
\hline Ex-smokers & $0(0 \%)$ & $5(4.2 \%)$ & 1.00 \\
\hline Current smokers & $5(71.4 \%)$ & $77(65.3 \%)$ & 1.00 \\
\hline Comorbidities, N (\%) & $1(14.3 \%)$ & $13(11.0 \%)$ & 1.00 \\
\hline COPD & $1(14.3 \%)$ & $0(0)$ & 0.06 \\
\hline Diabetes & $0(0)$ & $8(6.8)$ & 1.00 \\
\hline Hypertension & $0(0)$ & $8(6.8)$ & 1.00 \\
\hline Hepatitis B & $0(0)$ & $1(0.8)$ & 1.00 \\
\hline Fever, N (\%) & $4(57.1 \%)$ & $4(3.4 \%)$ & $<0.01$ \\
\hline \multicolumn{4}{|l|}{$\begin{array}{l}\text { Highest temperature during hospital stay, } \mathrm{N} \\
(\%)\end{array}$} \\
\hline$<37.5^{\circ} \mathrm{C}$ & $0(0 \%)$ & $2(1.7 \%)$ & 1.00 \\
\hline $37.5-38.0^{\circ} \mathrm{C}$ & $1(14.3 \%)$ & $2(1.7 \%)$ & 0.16 \\
\hline $38.1-39.0^{\circ} \mathrm{C}$ & $2(28.6 \%)$ & $0(0 \%)$ & $<0.01$ \\
\hline$>39.0^{\circ} \mathrm{C}$ & $1(14.3 \%)$ & $0(0 \%)$ & 0.06 \\
\hline $\mathrm{SpO}_{2}$, median (IQR) & $94(92-98)$ & $98(96-100)$ & 0.04 \\
\hline $\begin{array}{l}\text { Respiratory symptoms during admission, } \mathrm{N} \\
(\%)\end{array}$ & & & 1.00 \\
\hline Nasal congestion & $0(0 \%)$ & $0(0 \%)$ & \\
\hline Sore throat & $0(0 \%)$ & $0(0 \%)$ & \\
\hline Cough & $0(0 \%)$ & $2(1.7 \%)$ & \\
\hline
\end{tabular}

COPD, chronic obstructive pulmonary disease; $\mathrm{SpO}_{2}$, peripheral oxygen saturation, IQR, interquartile range. 


\begin{tabular}{|llll|}
\hline Variables & $\begin{array}{l}\text { Deteriorated } \\
\text { outcome } \\
(\mathbf{N}=7)\end{array}$ & $\begin{array}{l}\text { Improved } \\
\text { outcome } \\
(\mathbf{N}=118)\end{array}$ & $\begin{array}{c}P \\
\text { value }\end{array}$ \\
\hline Hemoptysis & $0(0 \%)$ & $0(0 \%)$ & \\
\hline Sputum production & $0(0 \%)$ & $0(0 \%)$ & 1.00 \\
\hline Shortness of breath & $2(28.6 \%)$ & $0(0 \%)$ & \\
\hline Other symptoms, $\mathrm{N}(\%)$ & & $0(0 \%)$ \\
\hline Fatigue & $0(0 \%)$ & $0(0 \%)$ \\
\hline Headache & $0(0 \%)$ & $0(0 \%)$ \\
\hline Nausea or vomiting & $0(0 \%)$ & $1(0.8 \%)$ \\
\hline Diarrhea & $0(0 \%)$ & \\
\hline $\begin{array}{l}\text { COPD, chronic obstructive pulmonary disease; } \mathrm{SpO}_{2}, \text { peripheral oxygen saturation, IQR, interquartile } \\
\text { range. }\end{array}$ & \\
\hline
\end{tabular}

Patients with improved outcomes were less likely to have abnormal chest CT findings and more likely to have had better pulse oximetry readings (Table 6). In addition, the blood leukocyte count in the deteriorated group was significantly higher than that in the improved group. 
Table 6

Comparison of chest CT findings and laboratory indices between COVID-19 patients with deteriorated or improved outcomes

\begin{tabular}{|c|c|c|c|}
\hline Variables & $\begin{array}{l}\text { Deteriorated outcome } \\
(\mathrm{N}=7)\end{array}$ & $\begin{array}{l}\text { Improved outcome } \\
(\mathrm{N}=118)\end{array}$ & $P$ value \\
\hline \multicolumn{4}{|l|}{ Chest computed tomography scan } \\
\hline Total abnormalities, N (\%) & $7(100 \%)$ & $78(66.1 \%)$ & 0.10 \\
\hline Ground-glass opacity & $7(100 \%)$ & $63(53.4 \%)$ & 0.02 \\
\hline Local patchy shadowing & $6(85.7 \%)$ & $12(10.2 \%)$ & $<0.001$ \\
\hline Bilateral patchy shadowing & $5(71.4 \%)$ & $5(4.2 \%)$ & $<0.001$ \\
\hline Interstitial abnormalities & $4(57.1 \%)$ & $0(0 \%)$ & $<0.001$ \\
\hline \multicolumn{4}{|l|}{ Laboratory findings } \\
\hline \multicolumn{4}{|l|}{ Complete blood counts } \\
\hline Leukocyte count & $7.9(6.6-9.5)$ & $6.3(5.2-7.3)$ & 0.04 \\
\hline$>10 \times 10^{9} / \mathrm{L}$ & $1(14.3 \%)$ & $1(0.8 \%)$ & 0.11 \\
\hline$<4 \times 10^{9} / \mathrm{L}$ & $1(14.3 \%)$ & $5(4.2 \%)$ & 0.30 \\
\hline Lymphocyte count & $2.2(1.6-2.8)$ & $2.0(1.6-2.3)$ & 0.51 \\
\hline$<1.5 \times 10^{9} / \mathrm{L}$ & $1(14.3 \%)$ & $20(17.0 \%)$ & 1.00 \\
\hline Platelet count & $224(157-303)$ & $215.5(172.5-261.0)$ & 0.76 \\
\hline$<150 \times 10^{9} / \mathrm{L}$ & $1(14.3 \%)$ & $14(11.9 \%)$ & 1.00 \\
\hline Hemoglobin level, g/L & $151(138-157)$ & $145(139.0-152.3)$ & 0.52 \\
\hline \multicolumn{4}{|l|}{ Hepatic and renal functions } \\
\hline AST > 40 U/L, N (\%) & $0(0 \%)$ & $14(11.9 \%)$ & 1.00 \\
\hline AST > 80 U/L, N (\%) & $0(0 \%)$ & $1(0.8 \%)$ & 1.00 \\
\hline ALT > $40 \mathrm{U} / \mathrm{L}, \mathrm{N}(\%)$ & $2(28.6 \%)$ & $48(40.7 \%)$ & 0.70 \\
\hline ALT > 80 U/L, N (\%) & $0(0 \%)$ & $16(13.6 \%)$ & 0.60 \\
\hline TB $17.1 \mu \mathrm{mol} / \mathrm{L}, \mathrm{N}(\%)$ & $0(0 \%)$ & $7(5.9 \%)$ & 1.00 \\
\hline Creatinine $\geq 133 \mu \mathrm{mol} / \mathrm{L}, \mathrm{N}(\%)$ & $0(0 \%)$ & $0(0 \%)$ & 1.00 \\
\hline
\end{tabular}

$\mathrm{CT}$, computed tomography; AST, aspartate aminotransferase; $\mathrm{ALT}$, alanine aminotransferase; $\mathrm{TB}$, total bilirubin; CRP, C-reactive protein; LDH, lactose dehydrogenase; CK, creatine kinase. 


\begin{tabular}{|c|c|c|c|}
\hline Variables & $\begin{array}{l}\text { Deteriorated outcome } \\
(\mathrm{N}=7)\end{array}$ & $\begin{array}{l}\text { Improved outcome } \\
(\mathrm{N}=118)\end{array}$ & $P$ value \\
\hline \multicolumn{4}{|l|}{ Blood biochemistry } \\
\hline Sodium, $\mathrm{mmol} / \mathrm{L}$ & $140(139.1-142.4)$ & $140.3(138.2-141.8)$ & 0.70 \\
\hline Potassium, mmol/L & $5.07(4.2-5.3)$ & $4.64(4.4-5.0)$ & 0.26 \\
\hline Chloride, mmol/L & $99.0(98.3-101.8)$ & $100.4(99.2-101.5)$ & 0.33 \\
\hline $\mathrm{CRP} \geq 10 \mathrm{mg} / \mathrm{L}, \mathrm{N}(\%)$ & $1(14.3 \%)$ & $9(7.6 \%)$ & 0.45 \\
\hline $\mathrm{LDH} \geq 250 \mathrm{U} / \mathrm{L}, \mathrm{N}(\%)$ & $0(0 \%)$ & $1(0.8 \%)$ & 1.00 \\
\hline$C K \geq 200 \mathrm{U} / \mathrm{L}, \mathrm{N}(\%)$ & $0(0 \%)$ & $4(3.4 \%)$ & 1.00 \\
\hline
\end{tabular}

\section{Discussion}

The ongoing pandemic of COVID-19 has imposed an enormous burden on the societies and individuals across the world[13-15]. Early studies have categorized the clinical spectrum of COVID-19 into mild, moderate, severe, or critical illness[4]. More than $80 \%$ of patients tend to recover with supportive care[9]. Fewer than $20 \%$ of patients require hospital admission for advanced healthcare. Previous studies have identified several risk factors for severe or critical illness; these include age, presence of comorbidities (such as hypertension, obesity, and diabetes), and certain laboratory indices[6, 8, 16-18]. However, young patients with no comorbid conditions who initially exhibit mild illness may rapidly develop severe or critical illness, and may even die[19]. Therefore, it is important to identify patients who have mild symptom at onset, but who are at a high-risk of developing critical illness. This can help optimize the use of healthcare resources and facilitate early intervention for high-risk patients.

COVID-19 may affect multiple organ systems in the body; however, it most commonly causes pneumonia[20]. Respiratory involvement is also the most common cause of death of patients with COVID-19[21]. Therefore, we first investigated the chest imaging findings in COVID-19 with mild clinical symptoms. Our results indicate that a sizable proportion of patients $(68.0 \%)$ with mild clinical symptoms may have abnormal chest imaging findings. Ground-glass opacity was the most common chest imaging abnormality. We further compared the differences between patients with improved or deteriorated outcomes during the stay at the mobile cabin hospital. Patients with deteriorated outcomes were significantly more likely to exhibit abnormal chest imaging findings than patients who had improved outcomes. Very few patients in our cohort had cough (2 patients) or shortness of breath (2 patients), even though a majority of patients $(85,68.0 \%)$ had abnormal chest imaging findings. This is consistent with a previous report that documented typical abnormal chest imaging findings in asymptomatic patients with COVID-19[22]. Therefore, we recommend chest imaging study of all patients with COVID-19, including 
those with mild symptoms. This may help identify patients who are at high risk of developing severe illness.

Chest imaging helps in the direct evaluation of pulmonary involvement in patients with COVID-19. However, chest imaging, especially CT scan, entails the risk of radiation exposure. It is also inconvenient to frequently perform repeat chest imaging. Peripheral oxygen saturation measurement is another method to indirectly assess the pulmonary ventilation and oxygenation status[23]. Moreover, it is a convenient and safe procedure. In our study, patients with deteriorated outcomes were significantly more likely to have low peripheral oxygen saturation than patients with improved outcomes. This suggested that monitoring of peripheral oxygen saturation is a useful method for identification of patients who may develop severe illness. Early initiation of oxygen therapy may reverse or save their lives.

In our study, patients with deteriorated outcomes were more likely to have moderately elevated temperature and elevated blood leukocyte count. Fever is the most common presenting feature of COVID19[24]. Studies have shown that SARS-CoV may attack leukocytes resulting in reduced leukocyte counts $[25,26]$. Further studies are required to assess the predictive value of body temperature and leukocyte count in COVID-19 patients with mild symptoms.

Several studies have identified comorbid conditions such as hypertension, diabetes, and chronic pulmonary disease as risk factors for severe and critical COVID-19 illness[6, 8, 16-18]. In our study, we did not observe any significant association of comorbidities with chest imaging findings or outcomes. Age was also found to be a risk factor for increased morbidity and mortality in COVID-19 patients[27]. In our study, older patients were more likely to have abnormal chest CT findings. However, age was not associated with the short-term outcome. We believe that the risk factors may differ depending on the initial presentation (mild or severe symptoms) of COVID-19 patients.

COVID-19 may impair the functioning of multiple organs and systems in the body. In addition to lung injury, liver and kidney are also frequently affected in these patients[28]. Some of the patients in our cohort exhibited mild elevation in liver enzyme levels; however, there was no significant difference between patients with deteriorated or improved outcomes in this respect. None of our patients showed any sign of kidney injury.

The single-center scope of our study and the relatively small sample size are some of the limitations of our study. We were only able to study adult male patients, since our mobile cabin hospital was exclusively dedicated for hospitalization of adult male COVID-19 patients. Lastly, this was an observational study; therefore, we could not control the treatment modalities, such as oxygen supplementation or administration of certain traditional Chinese medicines.

In summary, we observed an association of chest imaging findings, peripheral blood oxygen saturation, and body temperature with disease deterioration in adult male COVID-19 patients with mild clinical symptoms. Close monitoring of these indices may facilitate identification of patients who are high risk of 
developing severe or critical illness. This can help optimize the use of healthcare resources and facilitate early interventions to reduce morbidity and mortality in high-risk patients.

\section{Declarations}

\section{Acknowledgements}

We would like to express our thanks to the patients for their contributions to the study.

\section{Author Contributions}

Liping Peng and Dan Li conceived and designed the study. Bailing Yan and Yangyang Wang conducted the primary analysis and prepared the first draft of the manuscript. Lei Song and Jia Guo reviewed the draft for intellectual content. The corresponding authors Liping Peng and Dan Li had full access to all the data in the study and take responsibility for the integrity of the data and the accuracy of the data analysis.

\section{Funding}

This work was supported by National Natural Science Foundation of China for major research plan to Dan Li (2017ZX10302301-002) and Natural science foundation of jilin province to Liping Peng (JLSCZD2019-019). The funders had no role in the design and conduct of the study; collection, management, analysis, and interpretation of the data, review, or approval of the manuscript; and decision to submit the manuscript for publication. No authors have been paid to write this article by any pharmaceutical companies or agencies.

\section{Availability of data and materials}

All data generated or analysed during this study are included in this published article.

\section{Ethics approval and consent to participate}

Ethics Committee of the first hospital of Jilin university approved this study.

\section{Consent for publication}

Not applicable.

\section{Conflict of interest}

All authors declare that they have no competing interests.

\section{References}


1. Ge H, Wang X, Yuan X, Xiao G, Wang C, Deng T, Yuan Q, Xiao X: The epidemiology and clinical information about COVID-19. Eur J Clin Microbiol Infect Dis 2020.

2. Coronaviridae Study Group of the International Committee on Taxonomy of V: The species Severe acute respiratory syndrome-related coronavirus: classifying 2019-nCoV and naming it SARS-CoV-2. Nat Microbiol 2020, 5:536-544.

3. Cucinotta D, Vanelli M: WHO Declares COVID-19 a Pandemic. Acta Biomed 2020, 91:157-160.

4. Hassan SA, Sheikh FN, Jamal S, Ezeh JK, Akhtar A: Coronavirus (COVID-19): A Review of Clinical Features, Diagnosis, and Treatment. Cureus 2020, 12:e7355.

5. Huang C, Wang Y, Li X, Ren L, Zhao J, Hu Y, Zhang L, Fan G, Xu J, Gu X, et al: Clinical features of patients infected with 2019 novel coronavirus in Wuhan, China. Lancet 2020, 395:497-506.

6. Zhou Y, Zhang Z, Tian J, Xiong S: Risk factors associated with disease progression in a cohort of patients infected with the 2019 novel coronavirus. Ann Palliat Med 2020, 9:428-436.

7. Wang D, Hu B, Hu C, Zhu F, Liu X, Zhang J, Wang B, Xiang H, Cheng Z, Xiong Y, et al: Clinical Characteristics of 138 Hospitalized Patients With 2019 Novel Coronavirus-Infected Pneumonia in Wuhan, China. JAMA 2020.

8. Richardson S, Hirsch JS, Narasimhan M, Crawford JM, McGinn T, Davidson KW, and the Northwell CRC, Barnaby DP, Becker LB, Chelico JD, et al: Presenting Characteristics, Comorbidities, and Outcomes Among 5700 Patients Hospitalized With COVID-19 in the New York City Area. JAMA 2020.

9. Gandhi RT, Lynch JB, Del Rio C: Mild or Moderate Covid-19. N Engl J Med 2020.

10. Yao W, Wang X, Liu T: Critical role of Wuhan cabin hospital in controlling local COVID-19 pandemic. Infect Control Hosp Epidemiol 2020:1-6.

11. Sun C, Wu Q, Zhang C: Managing patients with COVID-19 infections: a first-hand experience from the Wuhan Mobile Cabin Hospital. Br J Gen Pract 2020, 70:229-230.

12. Shu L, Ji N, Chen X, Feng G: Ark of Life and Hope: the role of the Cabin Hospital in facing COVID-19. $J$ Hosp Infect 2020.

13. Acikgoz O, Gunay A: The early impact of the Covid-19 pandemic on the global and Turkish economy. Turk J Med Sci 2020, 50:520-526.

14. Ayittey FK, Ayittey MK, Chiwero NB, Kamasah JS, Dzuvor C: Economic impacts of Wuhan 2019-nCoV on China and the world. $J$ Med Viro/ 2020, 92:473-475.

15. Trilla A: One world, one health: The novel coronavirus COVID-19 epidemic. Med Clin (Engl Ed) 2020, 154:175-177.

16. Wu C, Chen X, Cai Y, Xia J, Zhou X, Xu S, Huang H, Zhang L, Zhou X, Du C, et al: Risk Factors Associated With Acute Respiratory Distress Syndrome and Death in Patients With Coronavirus Disease 2019 Pneumonia in Wuhan, China. JAMA Intern Med 2020.

17. Zhou F, Yu T, Du R, Fan G, Liu Y, Liu Z, Xiang J, Wang Y, Song B, Gu X, et al: Clinical course and risk factors for mortality of adult inpatients with COVID-19 in Wuhan, China: a retrospective cohort study. Lancet 2020, 395:1054-1062. 
18. Chen N, Zhou M, Dong X, Qu J, Gong F, Han Y, Qiu Y, Wang J, Liu Y, Wei Y, et al: Epidemiological and clinical characteristics of 99 cases of 2019 novel coronavirus pneumonia in Wuhan, China: a descriptive study. Lancet 2020, 395:507-513.

19. Team CC-R: Severe Outcomes Among Patients with Coronavirus Disease 2019 (COVID-19) - United States, February 12-March 16, 2020. MMWR Morb Mortal Wkly Rep 2020, 69:343-346.

20. Chen T, Wu D, Chen H, Yan W, Yang D, Chen G, Ma K, Xu D, Yu H, Wang H, et al: Clinical characteristics of 113 deceased patients with coronavirus disease 2019: retrospective study. BMJ 2020, 368:m1091.

21. Vincent JL, Taccone FS: Understanding pathways to death in patients with COVID-19. Lancet Respir Med 2020.

22. Meng $H$, Xiong R, He R, Lin W, Hao B, Zhang L, Lu Z, Shen X, Fan T, Jiang W, et al: CT imaging and clinical course of asymptomatic cases with COVID-19 pneumonia at admission in Wuhan, China. $J$ Infect 2020.

23. Smallwood CD, Walsh BK: Noninvasive Monitoring of Oxygen and Ventilation. Respir Care 2017, 62:751-764.

24. Guan WJ, Ni ZY, Hu Y, Liang WH, Ou CQ, He JX, Liu L, Shan H, Lei CL, Hui DSC, et al: Clinical Characteristics of Coronavirus Disease 2019 in China. N Engl J Med 2020, 382:1708-1720.

25. Liu Y, Liao W, Wan L, Xiang T, Zhang W: Correlation Between Relative Nasopharyngeal Virus RNA Load and Lymphocyte Count Disease Severity in Patients with COVID-19. Viral Immuno/ 2020.

26. Shi Y, Wang Y, Shao C, Huang J, Gan J, Huang X, Bucci E, Piacentini M, Ippolito G, Melino G: COVID19 infection: the perspectives on immune responses. Cell Death Differ 2020, 27:1451-1454.

27. Petretto DR, Pili R: Ageing and COVID-19: What is the Role for Elderly People? Geriatrics (Basel) 2020, 5.

28. Tian S, Xiong Y, Liu H, Niu L, Guo J, Liao M, Xiao SY: Pathological study of the 2019 novel coronavirus disease (COVID-19) through postmortem core biopsies. Mod Pathol 2020. 